\title{
Panorama Technique for 3D Animation movie, Design and Evaluating
}

\author{
Ola Mudher abd ${ }^{1}$, Dr. Karim Q. Hussein ${ }^{2}$ \\ ${ }^{1}$ UOITVnformatics Institute for Higher Studies/Baghdad-Iraq \\ ${ }^{2}$ Computer Science Dept./College of Science /Mustansiriya University/Baghdad-Iraq
}

\begin{abstract}
This paper presents an applied approach for Panorama 3D movies enhanced with visual sound effects. The case study that considered is IIPS@UOIT. Many selected S/W have been used to introduce the 3D Movie. 3D Animation is a modern technology in the field of the world of filmmaking and is considered the core of multimedia, where the vast majority of movies such as Hollywood movies that we see today, it was using $3 D$ technology. Where this technique is used in all the magazines, such as medical experiments, engineering, astronomy, planets and stars, to prove scientific theories, history, geography, etc., where they are building models or scenes or characters simulates reality and the movement of the viewer to the heart of the event. A three-dimensional film was made to (IIPS @ UOITC) to give it a future vision and published in the global sites such as YouTube, Facebook and Google earth. By using many specialized 3d software and cinematic tricks, with a focusing on movement, characters, lighting, cameras and final render.
\end{abstract}

Keywords: 3D animation, Modelling, Camera, Visual and Audio Effects, Skectup.

\section{Introduction}

A three-dimensional stereoscopic film (otherwise called three-dimensional film, 3D film or S3D film) is a movie that improves the illusion of depth perception, thus including a third measurement. The most widely recognized way to deal with the generation of $3 \mathrm{D}$ movies is gotten from stereoscopic photography. In it, a normal film camera framework is utilized to record the pictures as observed from two points of view (or PC created symbolism produces the two viewpoints in after generation.3D movies are not restricted to dramatic discharges; transmissions and direct-to-video movies have likewise consolidated comparable strategies, particularly since the approach of 3D TV and Blu-ray3D.

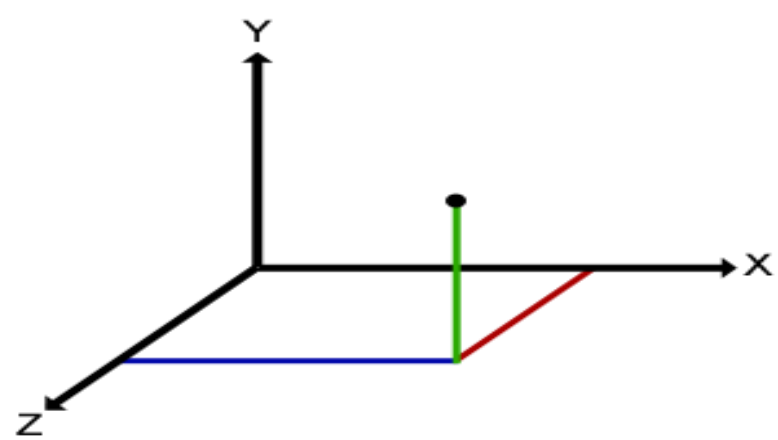

Figure .1 Basic 3D Technique

\section{Motivation towards research work}

Three-dimensional representation has been added to the viewing no limits enjoyment, this technology have been used in the cinema, where movies have become display to this wonderful technology, enabling the viewer feeling of great enjoyably when watching the movie characters of the three dimensions. Panorama 3D is a modern technology for Multimedia and it will take an active role in the market jobs.

This research aims is:

\section{Objective of Research}

1- Design a 3D model to the Informatics Institute for postgraduate studies in the University of information technology and communication, it considers one of the development aspects in Iraqi universities from using specialized software in this area.

2- Design high-quality three-dimensional movie HD1080pixel simulates reality and reflects the futuristic vision, where have benefits in:

- technological and scientific development. 
- Scientific to learn the secrets of this important and rapid technology.

- Educational to adds this new technology to Informatics Institute for postgraduate studies.

3 -Uploaded the 3D movie of IIPS to YouTube channel to make it more popular in High-quality format HD1080p.

Fourth: - product a film using many specialized software three-dimensional film making, cinematic tricks, with focusing on movement, characters, lighting, cameras and final rendering.

\section{Tools and requirements of 3D Panorama Animation Movie}

1.SketchUp program.

2.Adobe Photoshop CS5.

3.Adobe after Effects CS4.

4.Windows 764 bit operating system

5.4GB RAM (8 GB or more recommended

6. core i5 Processor

7.OpenGL graphics card supporting OpenGL 4.1 (dedicated GPU recommended)

8.Internet connection is required

9.Registration is required

\section{Stages of Implementing 3D movies Using Panorama Technique}

There are a series of stages for completing a 3D film deportation, where it is first configuring the main idea of the work and then modeling and construction phase to come later stage paint and Siding and then stirring the stage, one of the most important production phases of the project and the project's success depends greatly upon and then comes the lighting stage and then set up the camera and render a final stage and the production of the final video. In the next picture, simple diagram describes the main stages of the research. It is shown final target to upload the movie into Google Earth.

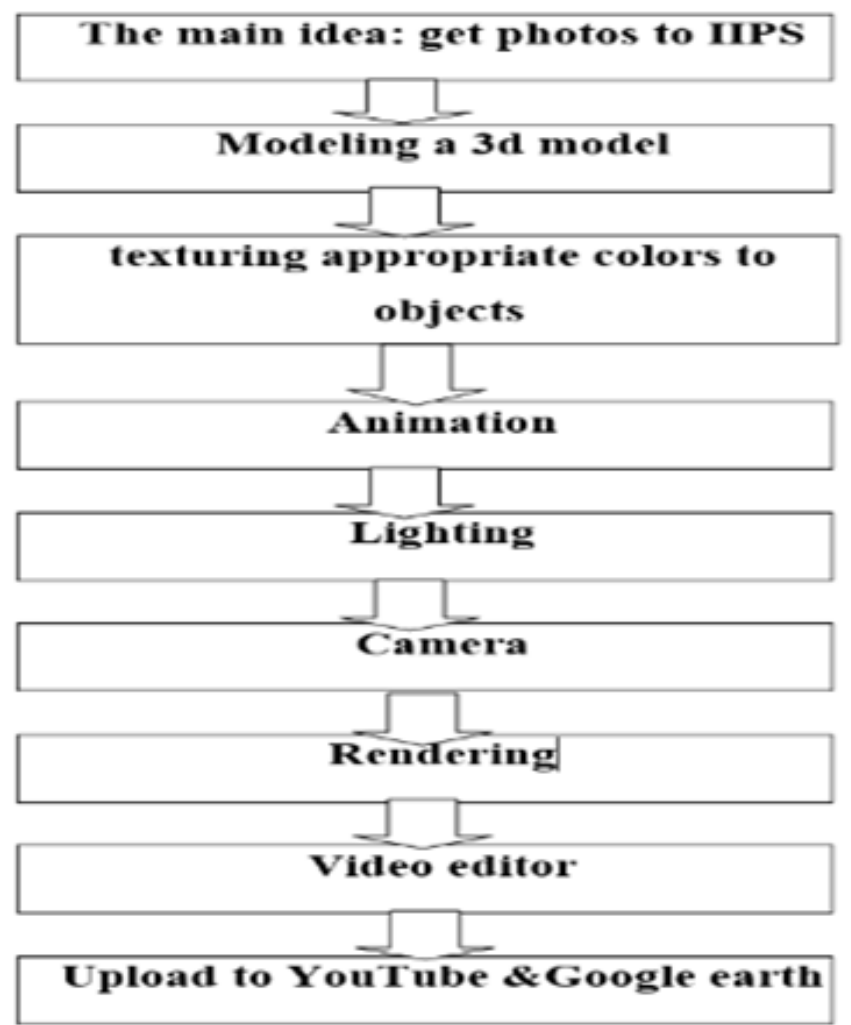

Figure .2 Basic 3D Stages Panorama Movie

5.1 The main idea:

Product A 3D movie, relying on Google earth and take pictures from different angles to university buildings and institute. in order to get locations and dimensions, sizes and coordinates to the university buildings. 


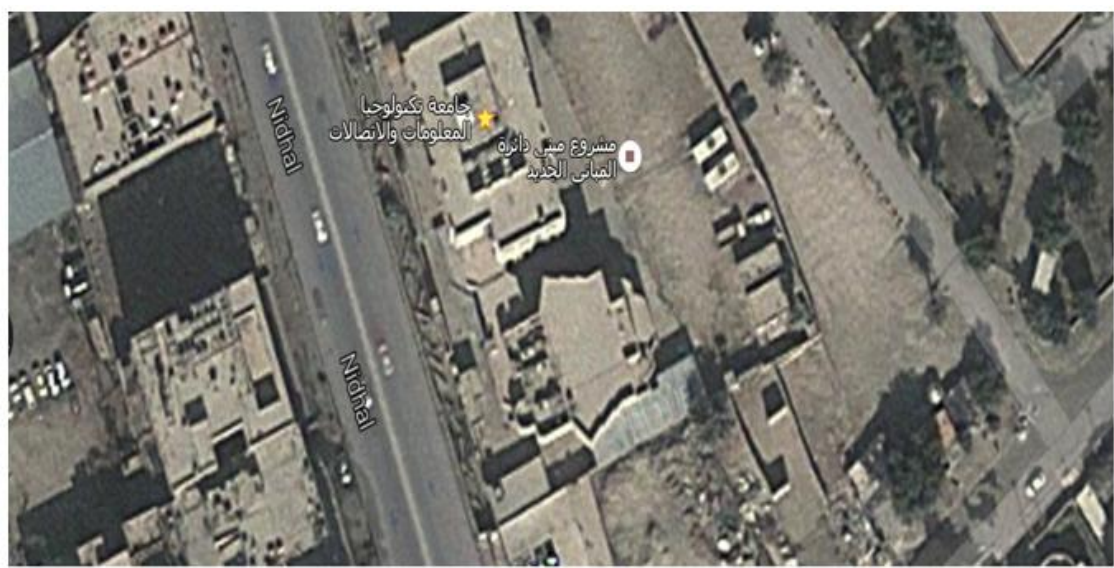

Figure .3 Main Idea

\subsection{The modelling}

The objects consider the basic in three-dimensional design, objects is a pre-design of geometric models are used to form more complex shapes or forms aggregates, In this stage the buildings and figures was designed that used in the project.

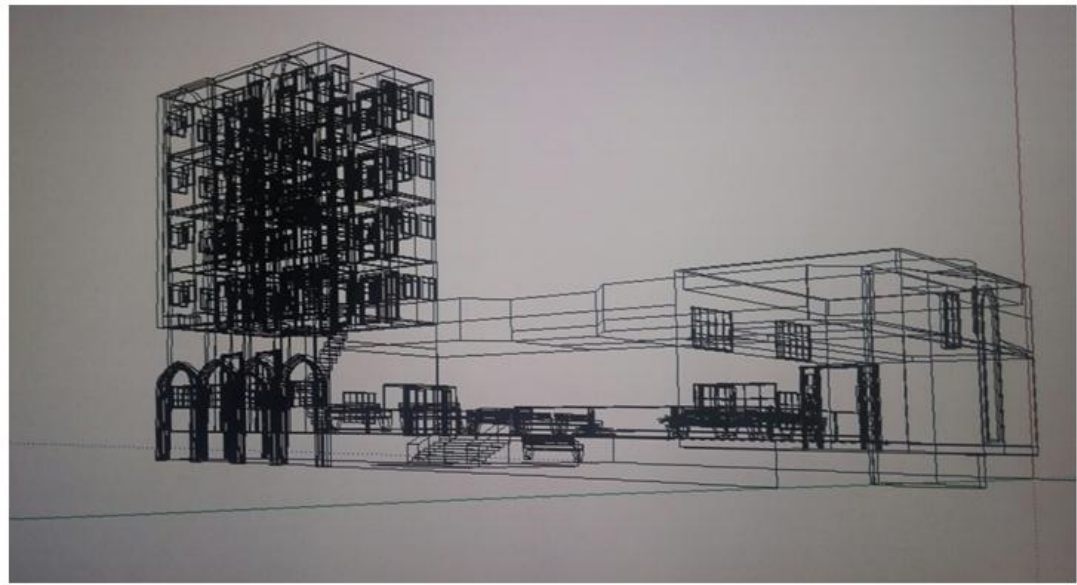

Figure .4 Model Stage

\subsection{The texture}

When you add any new element to the scene, it appears dark grey colour and this hypothetical case and can get realistic appearance of objects, depending mainly on the success of the designer in Siding process. It can make the surface shiny and apparently made of metal and can be made the same as the surface seems a rock, at this stage the appropriate paint the sky, walls, doors and floor like alabaster as well as clothes and colours appropriate for the characters designed observed was created.

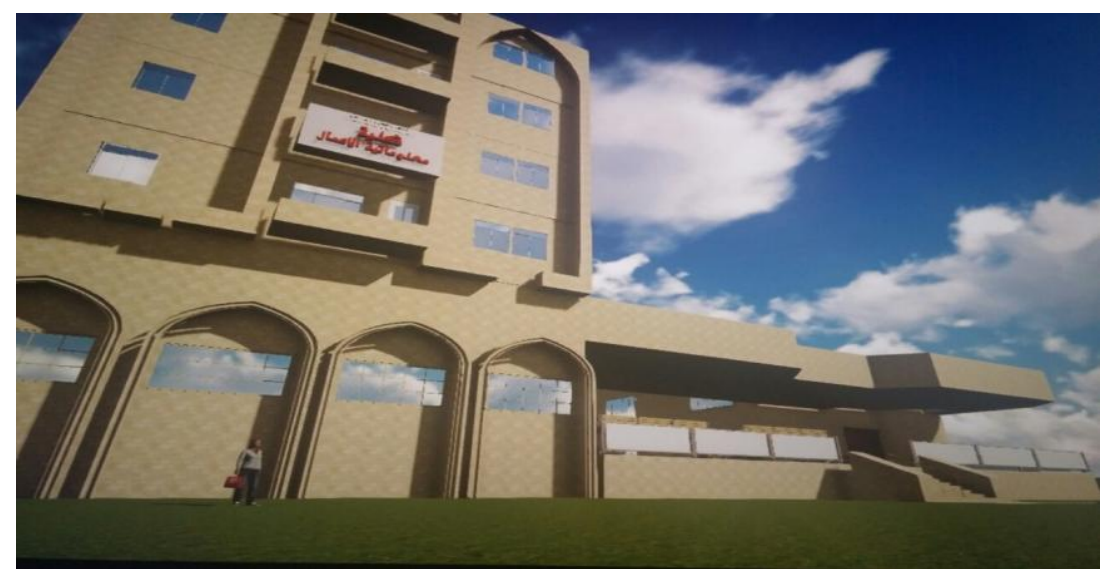

Figure .5 Texture Stage 


\subsection{Animation}

In this stage the necessary movement required was created (according to the subject of the scene) using UMION 3D visualization tool, and it makes the film more realistic and interesting, we see here the different movements of the character.

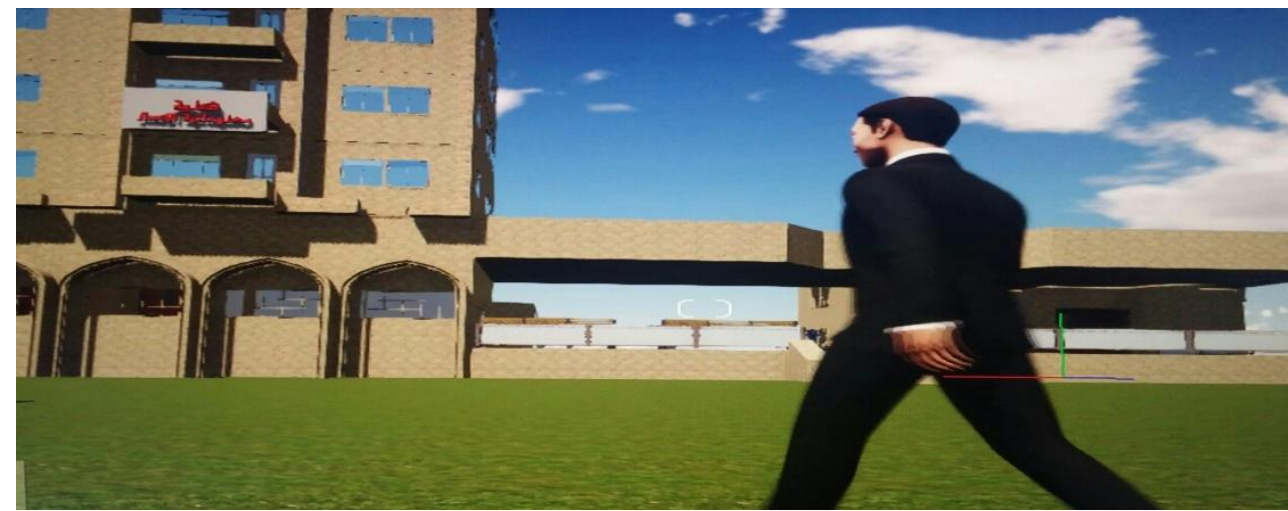

Figure .6 Animation Technique (3D)

\section{5 lighting}

Lighting and shadows are considered essential in the design of three-dimensional scenes, it gives the scene more realistic and consider it as an important factor in influencing the viewer a sense

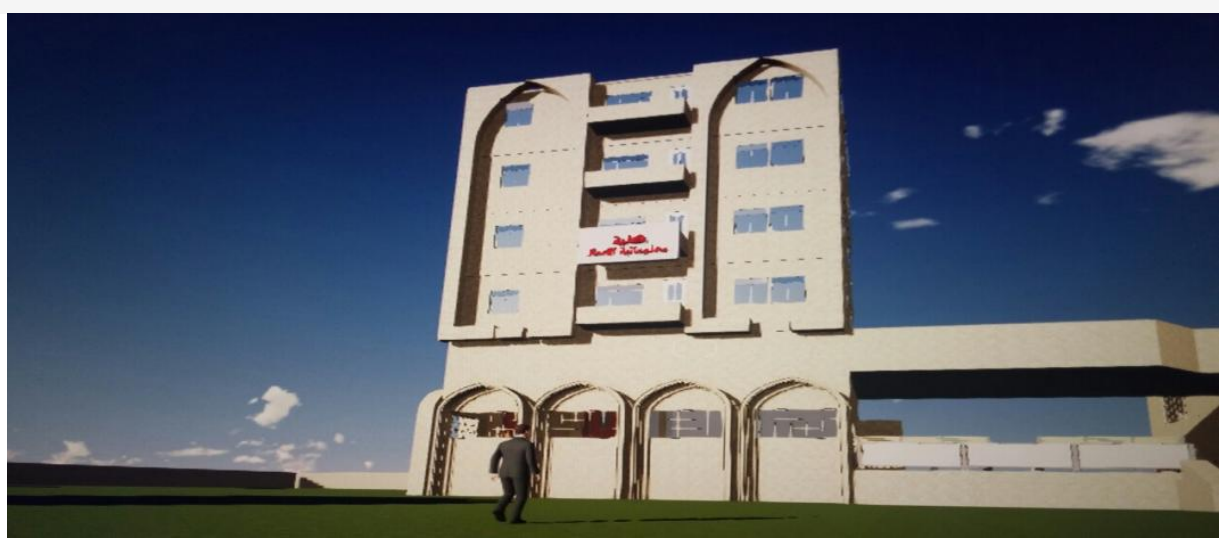

Figure .7 Lighting Sage

\subsection{Camera}

One of the important points that the designer must be taken into consideration in the production of three-film deportation - and before the imaging process is the angle of vision of the scene is done using one or more such as his command as needed and the scene to be filmed. Three-dimensional virtual programs provide cameras for any scene, which is the vision of the four ports, front, side, upper and unforeseen vision port.

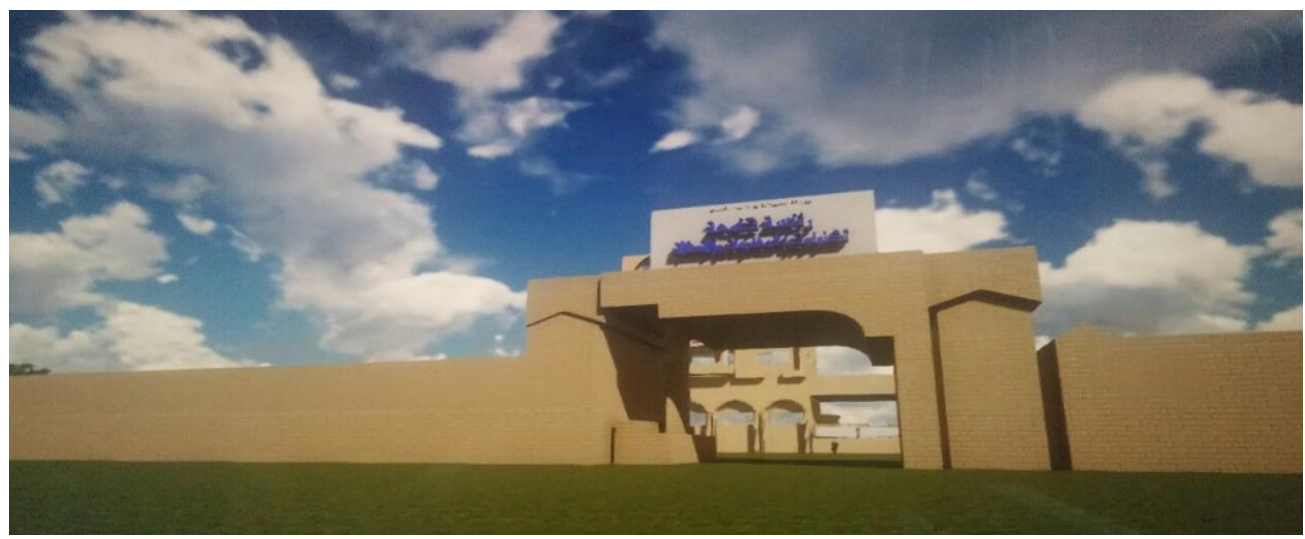

Figure .8 Camera Stage 


\subsection{Rendering}

In this stage Export and directed scene after the completion of all previous stages correctly. the following have been identified: -

- $\quad$ select format video with Avi.

- Custom size $1920 * 1080$.

- Select HD 1080 pixel.

- Select output range (number of frame).

- Render objects.

- Export video.

\subsection{Video Editor}

The following software's were used to edit the final video, audio, add visual and audio effects and cinematic tricks.

- Adobe After Effects CS4.

- Adobe Photoshop CS5.

\subsection{Uploading to YouTube and Google Earth}

This stage to define the institute and the university to the public and keep up with technological development

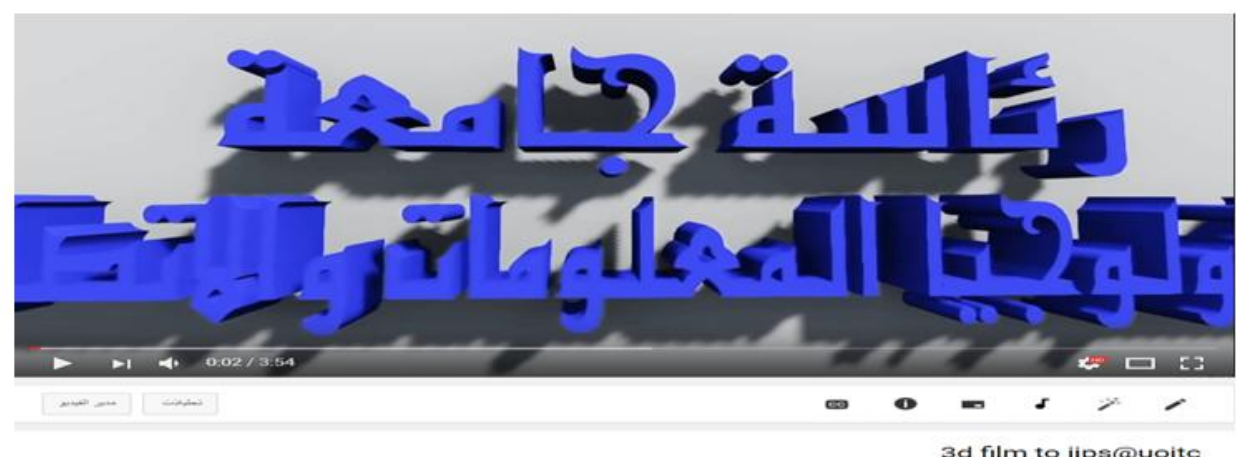

Figure.93D Panorama to IIPS@UOITC

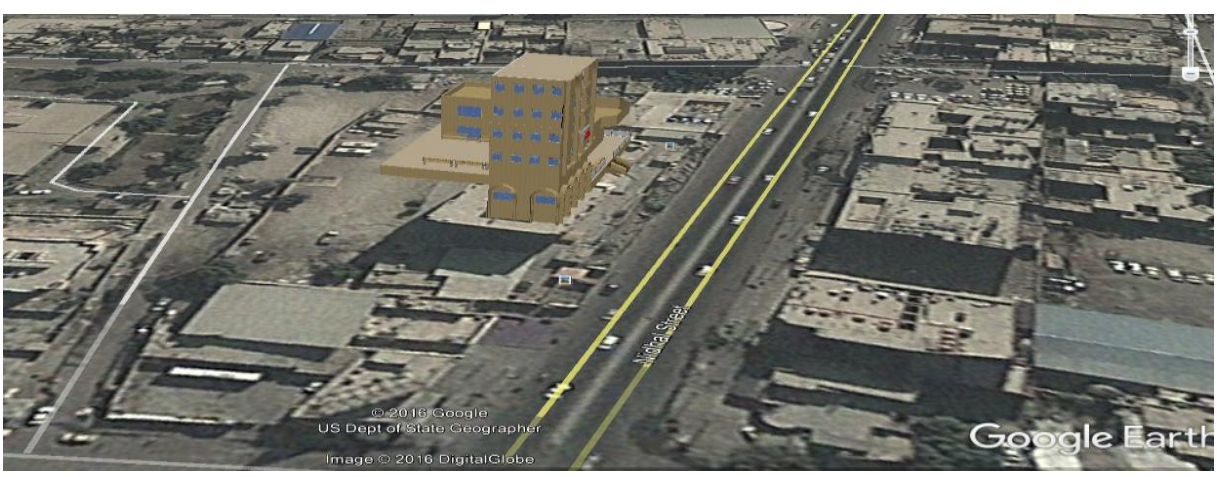

Figure .10 Uploading 3D Panorama for IIPS@UOITC to Google Earth

\section{Discussion}

The project rid the possibility of employing modern software to produce three-dimensional film to the Informatics Institute for postgraduate studies after a full understanding and perception of the project and what needs to be implemented.

\section{Conclusion}

i. Effects of color and sound play an active role on the quality of the film

The value of the sound is as important as the value of the picture, it adds a high technical and beauty value that makes the viewer interacts with the film a sense of high and closer to reality through which the director can make the viewer feel it inside the event.

ii. This Panorama represents integrated work to full view vision to IIPS, using proper Software.

iii. As final aspect of evaluation, The Panorama technique for 3D Movies gives an effective multimedia approach to edit desired 3D movies. 


\section{References}

[1]. T. Zizka, 3D Modeling. Cherry Lake Publishing, 2014.

[2]. Kapp, Karl M. O'Driscoll, Tony 'Learning in 3D : Adding a New Dimension to Enterprise Learning and Collaboration ' , December 2009.

[3]. Meng Li, Xin Yuan Huang, Tie Jun Zheng 'Application of 3D Animation Technology in Movie Art Design', Advanced Materials Research, Vol. 267, June 2011.

[4]. Waiyawat Saitum*, Cee Triamornpan, Nuttapon Thitichaiyongkit, Nuttawut Tipamornwiwat, '3D Animation Silent Movie Entitled "The Seed": Research for Level of Understanding Compare between Male and Female ' Department of Computer and Information Technology, Faculty of Industrial Education and Technology, KMUTT, Bangkok, Thailand, 2014.

[5]. Surendra Pal Singh, Kamal Jain, V. Ravibabu Mandla, 'Image Based 3D Modeling of Campus“ Geomatics Engineering Section, Department of Civil Engineering, Indian Institute of Technology, Roorkee, Uttarakhand, India, ISSN: 2348-5124, 2013.

[6]. Jeffrey Todd, 'The history of 3D technology',September 25, 2010.

[7]. Guenter POMASKA, 'Compiling Stereoscopic 3D Animations from 2D Video Sequences and Virtual Computer Models Exploiting Open Source Software', University of Applied Sciences Bielefeld, Faculty of Architecture and Civil Engineering, Germany.

[8]. F. Teubl,C. S. Kurashima ,M. C. Cabral,R. D. Lopes,J. C. Anacleto, M. K. Zuffo, 'An interactive perspective-corrected spherical 3D display“ „Fed. Univ. of Sao Carlos, Sao Carlos, Brazil, Published in: 3DTV-Conference: The True Vision - Capture, Transmission and Display of 3D Video (3DTV-CON), 2014, Date of Conference: 2-4 July 2014,Date Added to IEEE Xplore: 11 August 2014.

[9]. Maengsob JI , 'Image display apparatus, 3d glasses, and method for operating the image display apparatus` January,2011.

[10]. David H. Eberly, '3D Game Engine Design: A Practical Approach to Real-Time Computer Graphics` 2006 